\begin{tabular}{|c|l|}
\hline Title & Regularization of Inverse Problems in Reinforced Concrete Fracture \\
\hline Author(s) & Nazmul, I. M.; Matsumoto, T. \\
\hline Citation & $\begin{array}{l}\text { Journal of Engineering Mechanics, 134(10), 811-819 } \\
\text { https://doi.org/10.1061/ASCE)0733-9399(2008)134:10(811) }\end{array}$ \\
\hline Issue Date & 2008_10 \\
\hline Doc URL & http://hdl.handle.net/2115/48127 \\
\hline Type & article(author version) \\
\hline File Information & JEM134_10_811-819.pdf \\
\hline
\end{tabular}

Instructions for use 


\title{
Regularization of inverse problems in reinforced
}

\section{concrete fracture}

\section{By I. M. Nazmul ${ }^{1}$ and T. Matsumoto ${ }^{2}$}

\begin{abstract}
Reinforced concrete (RC) beams with flexural cracks are simulated by the bridged crack model. The weight function method of determining stress intensity factors has been followed to derive a transformation between the crack bridging force (the rebar force) and the crack opening displacements (CODs). The matrix of the transformation is then approximated by its finite difference equivalent within finite dimensional vector spaces. Direct problem of the transformation solves for CODs, which require a known rebar force. Alternatively, the inverse problem works out the rebar force from known CODs. But, the inverse transformations of such convolution type integral equations become ill-posed if input CODs are perturbed. The Tikhonov regularization method is followed in its numerical form to regularize the linear ill-posed inverse problem. Restoration of mathematical stability and consistency are demonstrated by specific
\end{abstract}

1. Assistant Professor, Department of Civil Engineering, Presidency University. Plot 11A, Road 92, Gulshan 2, Dhaka 1212, Bangladesh. E mail: islammn@presidency.edu.bd. Phone: 880-2-8857617 (ext. 501). Fax: 880-28831184.

2. Associate Professor, Division of Built Environment, Graduate School of Engineering, Hokkaido University, Kita 13, Nishi 8, Kita-ku, Sapporo 060-8628, Japan. E mail: takashim@eng.hokudai.ac.jp Phone: +81-11-706-6171 Fax: +81$11-706-6172$. 
examples, where the results of the direct and the corresponding inverse problem are cross-checked. Results of the direct problem (i.e. the analytical CODs) are deliberately perturbed by adding machine generated random numbers of a certain width. The inverse problems are solved with these CODs to simulate practical situations, where measured CODs data will inevitably be noisy. Computations reveal that the inverse analysis of CODs satisfactorily determines the rebar force without cross-section information.

CE Database subject headings: Concrete; cracking; fracture mechanics; transformations; reinforcing steels; force.

\section{Introduction}

In reinforced concrete (RC) structures, estimation of the crack bridging force by the reinforcing steels from surface crack opening displacements (CODs) is the inverse problem of a fracture mechanics based transformation. The transformation is an integral transform derived by following the weight function method of determining stress intensity factors. The direct problem of the transformation solves for CODs with known applied and reactive forces, and the inverse problem works out the crack bridging force from known CODs; either analytically estimated or measured on site. Since practically measured CODs are susceptible to data perturbation, the inverse problem becomes illposed and needs a regularization method to obtain a unique and stable solution. Direct problems of similar fracture mechanics based transformation in context of many engineering materials of various geometry and loading conditions were covered by 
Marshall et al. 1985, Marshall and Cox 1987, Cox and Marshall 1991a, McMeeking and Evans 1990, Buchanan et al. 1997, Fett et al. 1996 and Kitsutaka 1997. These direct problems were solved for CODs in fibrous composites and quasi-brittle materials (e.g., concrete, rocks, ceramics) assuming continuous bridging law functions ( $p-u$ relation, $p$ being the crack bridging force and $u$ the CODs). This paper addresses both the direct and the inverse problems pertinent to $\mathrm{RC}$ beams, where no $p-u$ relation exists due to complex state of stresses in the proximity of deformed bar ribs and discreteness of the bridging forces.

In this paper, the discrete rebar forces that tend to close the crack at rebar locations are simulated by unit step functions and are determined from CODs by the Tikhonov regularization method of the theory of inverse problems. Usually, rebar forces are computed by transformed $\mathrm{RC}$ section analysis equilibrating the moment of internal flexural stresses to the acting bending moment in the cross-section. Fracture mechanics based models using the weight function method are also available. Carpinteri (1984) simulated a cracked RC beam by Single Edge Notched (SEN) fracture specimen to estimate the rebar force by rotation congruence condition. The basic model was further extended to cyclic loading by Carpinteri and Carpinteri (1984), to the failure behavior of RC beams by Bosco and Carpinteri (1992), and to the constitutive flexural behavior of brittle-matrix composites by Carpinteri and Massabo (1997). These methods require the cross-section of the beam known explicitly beforehand, whereas a method exploiting CODs to determine the rebar force is still scarce. This paper adopts the bridged crack model to simulate cracked RC beams and utilizes the fracture mechanics concepts to 
relate the rebar force with CODs. Computations reported in this paper show that the rebar force may be determined from CODs without cross-section information, even when the practically measured CODs are noisy. At the same time, distribution of the rebar force along the crack indicates the location of rebars inside bulk concrete and their diameters, spacings, clear covers, etc.; the smaller the error, the better the approximation.

Cox and Marshall (1991b) followed the Tikhonov regularization method to solve an illposed inverse problem during the determination of the bridging law in continuously aligned fiber composites, and Massabo et al. (1998) followed the same method in characterizing the bridging mechanisms developed across delamination cracks by through-the-thickness reinforcements under mode II loading. In both cases, assumption of a continuous crack bridging force facilitated to expand $p(u)$ (or, $p(x), x$ is the location along the crack) by continuous orthogonal basis (Legendre polynomials, in their cases) within the closed intervals of $u$, giving way to adopt analytical procedures in regularization. But the discrete rebar force bridging the crack in $\mathrm{RC}$ do not have an analytical $p(u)$ relation within the closed interval of minimum and maximum CODs $\left[u^{\min }\right.$, $\left.u^{\max }\right]$, justifying the shift to numerical methods to solve the current ill-posed inverse problem.

This paper starts with the integral transform for cracked RC beams, and solves it both as a direct problem and as an inverse problem considering that the transformation is compact between inner-product spaces. The direct problem, which requires a known rebar force, is solved for the analytical CODs. To input into the direct problem, the rebar 
force may be calculated separately, e. g., by the transformed RC beam section analysis. Random numbers of a certain width are added to the analytical CODs to simulate the possible perturbation in the practically measured CODs. With these perturbed (or noisy) CODs, a finite dimensional approximation of the ill-posed inverse problem is introduced, where the extremals of the Tikhonov functional is determined in a finely discretized grid. The regularization parameter is chosen following the generalized discrepancy principle and fluctuations of results in different topological spaces are tested. The results of the inverse problem (i.e. the rebar force) are presented for different levels of noise in the CODs data.

The objective of this paper is to develop a method to determine the statically indeterminate rebar force from CODs, without making use of any cross-section parameters such as clear cover, reinforcement ratio, etc. Such a method will be a tool in maintenance engineering for nondestructive tests of existing structures for the cases where (1) original detailing is inadequate or unavailable and (2) damages (e.g., corrosion) or maintenance works (underlay, overlay) have altered the cross-section significantly.

\section{Basic Assumptions}

$\mathrm{RC}$ beams in pure flexure are idealized in Fig. 1 with a crack of length $a$ at the mid-span. The crack has already passed through all rebar layers along the total depth of the beam, $b$. The reactive rebar forces $F_{1}, F_{2}$ and $F_{3}$ tend to impede the opening due to active bending stress, $\sigma(x)$ on the crack plane, $x$. Such a single dominant crack at the mid-span resembles an SEN fracture specimen, and a zero or negligible shear at the crack plane ensures mode 
I fracture. Furthermore, a two dimensional analysis may be exploited since the crack is through-the-thickness, where relevant quantities are applicable to unit thickness of the beam. If the beam span is large compared to other dimensions, the weight function of an SEN specimen with infinite length and finite width is applicable.

Linear elastic material response is assumed for both concrete and steel focusing on maintenance of existing structures under service loading. Crumbling and crushing of concrete at the crack surfaces are taken into account by a discrete crack approach assuming that the crack is a finite thickness thin-layer discontinuity while the bulk concrete is a continuum. It is further assumed that a perfect and total force transfer occurs at steel-concrete interface into both sides of the crack. These assumptions are generally made in constitutive modeling of RC by fracture mechanics (e. g., Ben Romdhane and Ulm 2002) and the onset of cracking is manifested by the fracture toughness of concrete, $K_{I C}$ (Carpinteri, 1984, Bosco and Carpinteri 1992, Carinteri and Massabo 1997). The applicability of a single Linear Elastic Fracture Mechanics (LEFM) parameter is supported for concrete if a correct stress intensity factor calibration is available for specimens large enough compared to the crack length and/or aggregate size (Saouma et al. 1982). Since concrete fracture is characterized by extensive micro-cracking, surface roughness and three dimensional uneven apertures, application of LEFM needs a justification. Various models exist in the literature pertinent to cracking in materials with a relatively large fracture process zone ahead of the crack. A historical review of all relevant researches is available in Bazant (2002) with adaptation of LEFM models, viz. by an equivalent LEFM crack length, modification of stress intensity factor for size effect, 
etc. In the current analysis, the criterion for crack advance in the bridged crack model under monotonic loading is

$$
K_{\text {tip }}=K_{a}+K_{b}=K_{I C}
$$

where $K_{a}$ and $K_{b}$ are the stress intensity factors due to the external load and the rebar force respectively, while $K_{t i p}$ is the net stress intensity factor combining both effects.

\section{Fracture Mechanics Based Transformation}

With the assumptions described in the previous section, Castigliano's theorem is applied in the RC beam to derive CODs as

$$
u(x)=\frac{4}{E^{\prime}} \int_{x}^{a}\left[\int_{0}^{a^{\prime}} G\left(x^{\prime}, a^{\prime}, b\right)\left[\sigma\left(x^{\prime}\right)-f\left(x^{\prime}\right)\right] d x^{\prime}\right] G\left(x, a^{\prime}, b\right) d a^{\prime}
$$

where $E^{\prime}=E_{c}$ for plane stress and $E^{\prime}=E_{c} /\left(1-v^{2}\right)$ for plane strain respectively (Cox and Marshall 1991a). $E_{c}$ is the Young's modulus of concrete, $v$ the Poisson ratio and $G(x, a$, $b$ ) is the weight function to determine a stress intensity factor. Standard forms of weight functions for a large variety of geometry are available in the stress intensity factor handbooks. The weight function for an SEN specimen of infinite length and finite width is (Tada et al.1985)

$$
G(x, a, b)=\frac{1}{\sqrt{\pi a}} \frac{g(x / a, a / b)}{\left(1-x^{2} / a^{2}\right)^{1 / 2}(1-a / b)^{3 / 2}}
$$

where $g(x / a, a / b)=g(x / a, \xi)$ is given in Appendix A. 
The mechanics of a deformed bar pull-out from concrete is complicated due to interlocking of rebar ribs into bulk concrete along with complex conical stress patterns for secondary cracking (Goto 1971). In the current model, pull-out load is assumed linearly proportional to the acting bending moment until steel yielding. Post-yielding behaviors are not considered focusing on the service loading range, where acting bending moments do not exceed the nominal moment capacity. Within this range, variation of the rebar force along the crack is simulated (Fig. 2) by unit step functions as

$$
f(x)=\sum_{i=1}^{r} f_{i}\left[H\left(x-h_{i}\right)-H\left(x-h_{i}-d_{b i}\right)\right]
$$

where, by $f_{i}=\left(F_{i} / d_{b i}\right)$, the rebar force $F_{i}$ in the $i$-th layer is distributed within the diameter of rebars $d_{b i}$ of that layer, $r$ is the total number of rebar layers in the cross-section and $h_{i}$ the clear distance of a layer from the bottom face. The rebar force depends on CODs according to the material constitutive law and on the relative displacement (slip) between steel and concrete at the interface, which in turn, depends on the interfacial shear strength and the mechanical interlocking of rebar ribs into concrete. Crack bridging force contributing from strain softening of concrete is neglected for its insignificant role compared to the rebar force.

The net crack opening estimated by Eq. (2) is thought composed of two effects (Fett et al. 1996). First, the crack is opened due to applied load with the profile $u_{a}(x)$ and second, the reactive rebar force closes the crack by $u_{b}(x)$.

$$
u(x)=u_{a}(x)-u_{b}(x)
$$

where 


$$
u_{a}(x)=\frac{4}{E^{\prime}} \int_{x}^{a}\left[\int_{0}^{a^{\prime}} G\left(x^{\prime}, a^{\prime}, b\right) \sigma\left(x^{\prime}\right) d x^{\prime}\right] G\left(x, a^{\prime}, b\right) d a^{\prime}
$$

and with rearrangements

$$
u_{b}(x)=\frac{4}{E^{\prime}} \int_{x}^{a}\left[\int_{0}^{a^{\prime}} G\left(x^{\prime}, a^{\prime}, b\right) f\left(x^{\prime}\right) d x^{\prime}\right] G\left(x, a^{\prime}, b\right) d a^{\prime}=u_{a}(x)-u(x) .
$$

Eq. (7) may be considered a linear transformation $T: Z \rightarrow U$ between properly equipped linear vector spaces $Z$ and $U$ in the real numbers field, where $\mathbf{f} \in Z$ and $\mathbf{u}_{\mathbf{b}} \in U$. Linearity of the transformation is supported by observing

$$
T\left(\lambda_{1} \mathbf{f}_{1}+\lambda_{2} \mathbf{f}_{2}\right)=\lambda_{1} T\left(\mathbf{f}_{1}\right)+\lambda_{2} T\left(\mathbf{f}_{2}\right)
$$

where $\lambda_{1}, \lambda_{2} \in R$ are scalars, $R$ being the field of real numbers.

The functions $u_{b}(x)$ formed by the vectors $\mathbf{u}_{\mathbf{b}} \in U$ are all real valued continuous functions in the closed interval $[0, a]$, so we assume $U \subset L_{2}[0, a]$ by defining the metric $\rho_{2}: U \times U \rightarrow R$ as

$$
\rho_{2}\left(\mathbf{u}_{\mathbf{b}_{1}}, \mathbf{u}_{\mathbf{b}_{2}}\right)=\left[\int_{0}^{a}\left|u_{b_{1}}(x)-u_{b_{2}}(x)\right|^{2} d x\right]^{\frac{1}{2}}
$$

from which the definitions of norm $\|\cdot\|_{L 2}: U \times U \rightarrow \mathcal{R}$ and inner product $(\cdot, \cdot): U \times U \rightarrow \mathbb{R}$ in $\mathrm{U} \subset L_{2}[0, a]$ are derived.

The Tikhonov regularization method requires that the topology of the domain of the transformation $D(T) \subset Z$ has to be assumed from a priori considerations. Although the 
rebar force is simulated by unit step functions, the locations of the steps are unknown without cross-section details in the inverse problem. For the sake of mathematical computations in the inverse problem, we assume that the exact solution $\mathbf{f}^{-}$of analytical CODs $\mathbf{u}^{-}$is continuous on $[0, a]$. A unique and stable solution is sought with this assumption and consistency of data from the results of the direct and the corresponding inverse problem will be tested. Lack of stability in the inverse problem might enforce to assume a stronger norm (Kirsch 1996). Another set of computations is presented in this connection in Appendix B assuming $D(T)=W_{2}{ }^{1}[0, a]$; the vector space containing the functions along with their first derivatives that are square integrable over $[0, a]$. Currently assumed a priori condition enables assuming $\mathrm{Z} \subset L_{2}[0, a]$ which is a complete inner product space or Hilbert space (Michael and Herget 1981, Reddy 1986).

\section{Numerical Approximations}

The integral kernels of Eq. (2), (6) and (7) encounter singularities at $x=a$. So, the weight function is approximated by its finite difference equivalent in such a way that $|a-x|>0$. A suitable grid is chosen from the crack mouth to the crack tip as

$$
\left\{a_{k}, k=1, \ldots \ldots, m\right\} \text { and }\left\{x_{k}, k=1, \ldots \ldots, m\right\}
$$

where $x_{i}<a_{j}$ for $i \leq j$ with equal step interval $h_{x}$. Consequently, the weight function $G(x, a$,

$b$ ) is approximated with the matrix $g_{i j} \in \mathbf{G}$ for a certain specimen width as

$$
\left.\begin{array}{lc}
g_{i j}=G\left(x_{i}, a_{j}\right), & i=2, \ldots \ldots ., m-1 \\
g_{i j}=\frac{G\left(x_{i}, a_{j}\right)}{2}, & i=1, m
\end{array}\right\} j=1, \ldots, m .
$$


The approximated weight function should be real-valued, continuous and non-singular on

$$
\Pi=\left\{0 \leq a^{\prime} \leq a, 0 \leq x<a^{\prime}\right\}
$$

The matrix $\mathbf{G}$ is a lower triangular matrix for the choice of a grid stated in Eq. (10). Approximated weight function leads to an approximated transformation $T_{\beta}$ instead of $T$ defined by Eq. (7) with error level $\beta>0$ as

$$
\left\|T-T_{\beta}\right\| \leq \beta
$$

Both the transformations $\left(T\right.$ and $T_{\beta}$ ) are $L_{2}[0, a] \rightarrow L_{2}[0, a]$, hence withdrawn from being stated in Eq. (13). Inverse problems where the transformation itself is given approximately by another bounded linear operator are also regularizable for $\beta \rightarrow 0$ (Tikhonov et al. 1990). An example of this approximation with exact and approximated transformations are shown in Fig. 3 in terms of CODs profiles of a rectangular beam having three layers of rebars with a crack passing through $413 \mathrm{~mm}$ along the total depth of $600 \mathrm{~mm}$. Further detail of this beam is given in the numerical examples section.

CODs data should be measured at the grid points as

$$
\mathbf{u}:=\left\{u_{k}, k=1, \ldots \ldots, m\right\}
$$

the finer the grid, the better the accuracy in computation. Crack openings due to applied load, $u_{a}(x)$ in Eq. (6) are deterministic and are computed for any loading magnitude and distribution with desired accuracy as

$$
\mathbf{u}_{\mathbf{a}}:=\left\{u_{a_{k}}, k=1, \ldots \ldots, m\right\}
$$


and following Eq. (7), CODs $\mathbf{u}$ are subtracted from crack openings $\mathbf{u}_{\mathbf{a}}$ to get the crack closings by the rebar force. The vector $\mathbf{u}_{\mathbf{b}} \in \mathbb{R}(T) \subset U$ being computed at the grid points is

$$
\mathbf{u}_{\mathbf{b}}:=\left\{u_{b_{k}}, k=1, \ldots \ldots, m\right\}=\mathbf{u}_{\mathbf{a}}-\mathbf{u} .
$$

The rebar force $\mathbf{f} \in D(T)$ is computed in the inverse problem at the same grid points as

$$
\mathbf{f}:=\left\{f_{k}, k=1, \ldots \ldots, m\right\}
$$

\section{Inverse Analysis}

Eq. (7) defines a linear and bounded transformation $T: D(T) \subset Z \rightarrow U$ between Hilbert spaces $Z$ and $U$ as

$$
T \mathbf{f}=\mathbf{u}_{\mathrm{b}} \text {, with } \mathbf{f} \in Z \text { and } \mathbf{u}_{\mathbf{b}} \in U
$$

Solution of Eq. (18), presumably $\mathbf{f}=T^{-1} \mathbf{u}_{\mathbf{b}}$, is not stable if data $\mathbf{u}_{\mathbf{b}}$ is perturbed as $\mathbf{u}_{\mathbf{b}}{ }^{\delta}$ with an error level $\delta$. The inverse operator $T^{1}$ may not exist at all since the perturbed $\mathbf{u}_{\mathbf{b}}{ }^{\delta}$ does not necessarily belong to the domain of $T^{1}$. Even if $\mathbf{u}_{\mathbf{b}}{ }^{\delta} \in \mathrm{D}\left(T^{-1}\right)$, the metric (or distance) $\rho_{Z}\left(T^{1}\left(\mathbf{u}_{\mathbf{b}}{ }^{\delta}\right), \mathbf{f}^{-}\right)$may not tend to zero as $\delta \rightarrow 0$, where $\mathbf{f}^{-}$is the analytical solution for the analytical CODs data as

$$
T \mathbf{f}^{-}=\mathbf{u}_{\mathbf{b}}^{-}
$$

So, the problem is incorrectly posed or ill-posed. We have to approximately compute the abstract transformation $T^{-1}$ for an imprecisely given argument $\mathbf{u}_{\mathbf{b}}{ }^{\delta}$, as well as for an approximately given transformation $T_{\beta}$ with error $\beta$. Hence, a pair $\left(\mathbf{u}_{\mathbf{b}}{ }^{\delta}, \delta\right)$ is given such that 


$$
\rho_{U}\left(\mathbf{u}_{\mathbf{b}}^{\delta}, \mathbf{u}_{\mathbf{b}}^{-}\right) \leq \delta
$$

Solving an ill-posed problem needs regularizing algorithm which minimizes the discrepancy. There may be a family of regularizing operators depending on error set $\eta=$ $(\delta, \beta)$ such that

$$
\lim _{\delta \rightarrow 0} \sup _{\mathbf{u}_{\mathbf{b}}^{\mathbf{\delta}} \in U} \rho_{Z}\left(R_{\eta}\left(\mathbf{u}_{\mathbf{b}}^{\mathbf{\delta}}\right), T^{-1}\left(\mathbf{u}_{\mathbf{b}}^{-}\right)\right) \rightarrow 0
$$

but we adopt the following smoothing functional proposed in the Tikhonov regularization method and determine the minimal of this functional for our transformation in Eq. (7)

$$
M^{\alpha}[\mathbf{f}]=\left\|T_{\beta} \mathbf{f}-\mathbf{u}_{\mathbf{b}}^{\delta}\right\|_{U}^{2}+\alpha\|\mathbf{f}\|_{Z}^{2}
$$

where $\alpha$ is the regularization parameter.

Numerical solution of ill-posed problems approximates the initial infinite-dimensional problem to a finite dimensional one, for which numerical algorithm and computer programs can be developed. To ensure better convergence of the extremals of the Tikhonov functional with those of the finite dimensional approximation, the dimension of the finite dimensional approximation should increase unboundedly (Tikhonov et al. 1990). We choose a sufficiently large dimension so that the error in the approximation is substantially small. Thus the following Tikhonov functional, obtained by substituting Eq. (7) into Eq. (22)

$$
M[f(x)]=\int_{0}^{a}\left[\frac{4}{E^{\prime}} \int_{x}^{a}\left\{\int_{0}^{a^{\prime}} G_{h}\left(x^{\prime}, a^{\prime}\right) f\left(x^{\prime}\right) d x^{\prime}\right\} G_{h}(x, a) d a^{\prime}-u_{b}^{\delta}(x)\right]^{2} d x+\alpha \int_{0}^{a}|f(x)|^{2} d x
$$

is approximated as 


$$
\hat{M}^{\alpha}[\mathbf{f}]=\sum_{k=1}^{m}\left[\sum_{j=1}^{m} g_{k j}\left(\sum_{i=1}^{m} g_{i j} f_{i}\right) h_{x}-u_{b_{k}}^{\delta}\right]^{2} h_{x}+\alpha \sum_{k=1}^{p} f_{k}^{2}
$$

Variations of Eq. (24) with respect to $f_{k}$ lead to the following $m$ number of equations

$$
h_{x}^{2} \sum_{j=1}^{m} \sum_{k=1}^{m}\left(\sum_{i=1}^{m} g_{l i} g_{k i}\right)\left(\sum_{i=1}^{m} g_{j i} g_{k i}\right) f_{j}+\alpha h_{x} f_{l}=h_{x} \sum_{k=1}^{m}\left(\sum_{i=1}^{m} g_{l i} g_{k i}\right) u_{b_{k}}
$$

for $l=1, \ldots \ldots \ldots, m$. We solve this as the following system of equations

$$
\mathbf{B f}+\alpha \mathbf{C f}=\mathbf{v}
$$

where

$$
\begin{aligned}
& b_{l j}=h_{x} \sum_{k=1}^{m}\left(\sum_{i=1}^{m} g_{l i} g_{k i}\right)\left(\sum_{i=1}^{m} g_{j i} g_{k i}\right) \in \mathbf{B} \\
& v_{l}=\sum_{k=1}^{m}\left(\sum_{i=1}^{m} g_{l i} g_{k i}\right) u_{b_{k}}^{\delta} \in \mathbf{v} .
\end{aligned}
$$

The matrix $\mathbf{C}$ in Eq. (26) is an $m \times m$ identity matrix for the case of a same norm in both the domain and the range (as we considered $L_{2}[0, a] \rightarrow L_{2}[0, a]$ ). Different definitions of norms will make the solution more complicated without altering the results significantly as demonstrated in Appendix B, although it might be inevitable in some cases.

The choice of the regularization parameter $\alpha>0$ is made by following generalized discrepancy principle which consists of constructing a function $\alpha=\alpha(\eta)$ for errors $\eta=$ $(\delta, \beta)$ along with the condition $\left\|\mathbf{u}_{\mathbf{b}}{ }^{\delta}\right\|>\delta$. Computations reported in this paper took $\alpha>0$ as the zero of the following generalized discrepancy 


$$
\rho_{\eta}(\alpha)=\left\|T_{\beta} \mathbf{f}_{\boldsymbol{\eta}}^{\boldsymbol{a}}-\mathbf{u}_{\mathbf{b}}^{\delta}\right\|^{2}-\left(\delta+\beta\left\|\mathbf{f}_{\boldsymbol{\eta}}^{\boldsymbol{\alpha}}\right\|\right)^{2}
$$

We find the vector $\mathbf{f}$ from a family of approximate solutions $\mathbf{f}_{\eta}{ }^{\alpha}$, depending on the regularization strategies. For cases where no zero exists and $\rho_{\eta}(\alpha)>0$ for all $\alpha>0$, the solution is

$$
\mathbf{f}_{\boldsymbol{\eta}}=\lim _{\alpha \downarrow 0} \mathbf{f}_{\boldsymbol{\eta}}^{\alpha}
$$

\section{Solution Procedure}

Solution of the direct problem is not unstable if a known rebar force $f(x)$ is substituted into Eq. (2) from a separate computation. For example, the rebar force may be computed by balancing the moment of the couple generated by concrete compression and rebar tension in the cross-section with the applied bending moment (transformed section analysis) as

$$
F=\frac{M}{j d}
$$

where $j$ is the section parameter and $d$ is the effective depth of the beam. Small errors in the rebar force computation do not lead to large variations in the results of the direct problem (i. e. the CODs), since the transformation itself is a smoothing integral transform. But, the inverse transformation is the opposite of integration, where a small error in CODs may lead to errors greater than the worst case error, which in some cases tends to infinity (Kirsch 1996).

The direct problem of Eq. (2) computes the analytical CODs. If the basic assumptions, and the fracture mechanics based transformation are close to the reality, these analytical 
CODs should be close to the practically measured CODs on real concrete structures. Slight deviations originating from measuring technique and devices are not unexpected. The measurement errors, which inevitably perturb the CODs are simulated by adding random numbers to the analytical CODs. The machine generated random numbers may have any statistical distribution within a certain interval. For example, random numbers having a normal (Gaussian) distribution with a zero mean and a certain standard deviation may be chosen so as to invoke $0.1 \%$ to $10 \%$ errors in the analytical CODs. Thus the analytical CODs are converted to noisy CODs to enter into the inverse problem, where no cross-sectional information is required to determine the rebar force. The Euler equation in Eq. (26) is solved for the unknown vector $\mathbf{f}$ with a perturbed right hand side. Families of regularization strategies for different values of the regularization parameter are examined and the most appropriate one defined by Eq. (29) and Eq. (30) are taken. The computed rebar force is expected to match the previous one used in the direct problem computed by Eq. (31). It is observed that data with very low level of noise retrieves the expected rebar force and the results become inaccurate with the increase of error percentages.

\section{Numerical Examples}

The first set of computations reported in this section is relevant to the cross-section shown in Fig. 4. A singly reinforced RC beam with three layers of rebars into the tension side is considered where the clear distances between reinforcement layers are $20 \mathrm{~mm}$ with a bottom clear cover $30 \mathrm{~mm}$. Normal strength concrete with compressive strength 20 
MPa is coupled with Grade 400 steel rebars. The cross-section has a nominal moment capacity $M_{\mathrm{n}}=282 \mathrm{kN}-\mathrm{m}$ and mode I fracture condition is considered with zero or negligible shear at the crack plane.

\section{Direct problem}

The crack length at a certain load in monotonic loading is determined by the fracture condition given in Eq. (1). First, the net stress intensity factor $K_{t i p}$ is computed as the total effect of fracturing by the applied load, and shielding by the rebar force as

$$
K_{t i p}=K_{a}-K_{b}=\int_{0}^{a} G(x, a, b)[\sigma(x)-f(x)] d x .
$$

Choice of the weight function $G(x, a, b)$ stated in Appendix A for computing the stress intensity factor of RC beams deserves a scrutiny. A finite element analysis by ANSYS package is presented in Appendix C, where the stress intensity factors computed by Eq. (32) are compared with those by the finite element analysis. $\sigma(x)$ in Eq. (32) is the bending stress that would have existed on the crack plane if there was no crack. $\sigma(x)$ is caused by the applied load and has a linear profile as

$$
\sigma(x)=\sigma_{\max }\left(1-\frac{2 x}{b}\right)
$$

where, $\sigma_{\max }$ is the maximum stress at the top/bottom fiber of the cross-section. The total rebar force $F$ determined by Eq. (31) is distributed among the rebar layers as indicated in Eq. (4) considering a linear variation of strains in the cross-section with a zero strain at the neutral axis. 
Following Eq. (32), $K_{\text {tip }}$ for various levels of service bending moments are estimated and shown in Fig. 5. The crack tip at a certain load may be located as the point of intersection of a horizontal line through $K_{I C}$ with the $K_{\text {tip }}$ curve of that load from the right direction. Depending on specimen size, geometry and concrete compressive strength, critical stress intensity factor $K_{I C}$ for concrete varies between $6 \mathrm{~N} / \mathrm{mm}^{3 / 2}$ to $44 \mathrm{~N} / \mathrm{mm}^{3 / 2}$. Higgins and Bailey (1976) experimentally showed that concrete $K_{I C}$ reached a plateau value of 20 $\mathrm{N} / \mathrm{mm}^{3 / 2}$ for larger concrete specimens, and Lou et al. (2003) related concrete compressive strength with $K_{I C}$, which varied between $20 \mathrm{~N} / \mathrm{mm}^{3 / 2}$ to $26 \mathrm{~N} / \mathrm{mm}^{3 / 2}$ for normal strength concrete. This paper took $K_{I C}=20 \mathrm{~N} / \mathrm{mm}^{3 / 2}$ to demonstrate onset of cracking in concrete. Adaptation of LEFM models suggested by Bazant (2002) may be followed to alter computed stress intensity factors for size effects. Thus the crack length is determined and a suitable grid is chosen following Eq. (10). Finally Eq. (2) is directly solved yielding CODs profiles shown in Fig. 6.

\section{Inverse problem}

Analytical CODs $\mathbf{u}^{-}$are collected from a certain profile of Fig. 6 and made noisy CODs, $\mathbf{u}^{\delta}$ by adding random numbers to them. The errors in the noisy CODs are calculated in percentages as

$$
\operatorname{error}(\%)=\frac{\left\|\mathbf{u}^{-}-\mathbf{u}^{\boldsymbol{\delta}}\right\|}{\left\|\mathbf{u}^{-}\right\|} \times 100
$$

Consequently, $\mathbf{u}_{\mathbf{b}}$ is perturbed into $\mathbf{u}_{\mathbf{b}}{ }^{\delta}$, which are entered into Eq. (28), and Eq. (26) is solved for $\mathbf{f}$. Various percentages of errors in CODs yielded the rebar forces shown in Fig. 7(b) $-7(d)$, where Fig. 7(a) is the analytically computed rebar force by Eq. (31) used in 
the direct problem. The objective of the inverse problem is to recover an approximation of the stepped function of Fig. 7(a) from perturbed CODs. Accuracy in recovery is evident as Fig. 7(b) - 7(d) are compared with Fig. 7(a).

The second set of computations relevant to Fig. 8 is more specific. A rectangular crosssection RC beam with two layers of rebars is on a four-point loading. Loads, materials, cross-section and geometric properties are delineated on Fig. 8(a) - 8(b). Fig. 8(c) shows the rebar force computed by Eq. (31) for using in the direct problem, and Fig. 8(d) presents the relevant CODs profile; the result of the direct problem. The dotted line with circular markers in Fig. 8(d) shows the noisy CODs, after random numbers have been added to the analytical CODs. Data points are collected from the dotted curve and entered into the inverse problem. Two sets of noisy CODs having $1 \%$ and $5 \%$ data errors are entered into the inverse problem, and the rebar forces presented in Fig. 8(e) - 8(f) are obtained. Retrieval of the original rebar force of Fig. 8(c) is evident as it is compared with Fig. 8(e) - 8(f) along with the calculated values shown in the figure.

It is found that the rebar forces are determined with good accuracy within a moderate level of noise and the results lose accuracy with increased error percentages in CODs data. Excellent accuracy is observed for extreme noiseless data, where the results are capable of predicting the locations, diameters and layers of rebars. Further improvement of the results may be achieved by another direct analysis with the current rebar force from inverse analysis. But results changed very insignificantly in this case and it became stable after one cycle of iteration. 
For practical application, very sophisticated techniques should be followed to measure CODs. At the same time, instrumental and computational error levels should be defined accurately for a correct choice of the regularizing parameter. Collections of surface CODs data are reported by using Scanning Electron Microscope (SEM), Laser Interferometric Displacement Gauge (IDG) (Buchanan et al. 1997, Rodel et al. 1990, Studer et al. 2002) where noise level is limited within several microns. A high resolution digital camera or a scanning microscope might be sufficient for massive concrete structures.

\section{Conclusion}

The Tikhonov regularization method has been exploited to compute the rebar force from noisy CODs. The Tikhonov functional relevant to a transformation of RC beam fracture is derived and approximated within finite dimensional vector spaces. Variation of the functional yielded the normal equation, solution of which are the discrete rebar forces. Thus a numerical method for the solution of the ill-posed inverse problem is presented.

The discrete rebar forces are simulated by unit step functions. Direct problems of the transformation are also solved and analytical CODs in RC beams are computed. Accuracy of the developed mathematical procedures is checked by consistency of results of the direct and the corresponding inverse problem. 
Effects of various percentages of errors in the CODs while computing the rebar force are tested. A clear picture of the cross-section may be obtained if errors in CODs are extremely small. Thus the presented method demonstrates its non-destructive nature in determining internal geometric condition and stress states from external CODs measurements.

Acknowledgement: This study was supported by the Program for Promoting Fundamental Transport Technology Research from the Japan Railway Construction, Transport and Technology Agency (JRTT).

\section{References}

Bazant, Z. P. (2002). "Concrete fracture models: testing and practice." Eng. Fract. Mech., 69(2), $165-205$.

Ben Romdhane M. R., and Ulm, F. -J. (2002). "Computational mechanics of the steel concrete interface." Int. J. Numer. Anal. Met., 26(2), 99-120.

Bosco, C., and Carpinteri, A. (1992). "Fracture behavior of beams cracked along reinforcement.” Theor. Appl. Fract. Mec., 17(1), 61-68.

Buchanan, D. J., John, R., and Johnson, D. A. (1997). "Determination of crack bridging stress from crack opening displacement profiles." Int. J. Fracture., 87(2), 101-117.

Carpinteri, A. (1984). "Stability of fracturing process in RC beams." J. Struct. Eng.ASCE, 110(3), 544-558. 
Carpinteri, A., and Carpinteri, A. (1984). "Hysteretic behavior of RC beams." J. Struct. Eng.- ASCE, 110(9), 2073-2084.

Carpinteri, A., and Massabo, R. (1997). "Continuous vs discontinuous bridged crack model for fiber reinforced materials in flexure.” Int. J. Solids. Struct., 34(18), 2321 2338.

Cox, B. N., and Marshall, D. B. (1991a). "Stable and unstable solutions for bridged crack in various specimens." Acta. Metall. Mater., 39(4), 579-589.

Cox, B. N. and Marshall, D. B. (1991b). "The determination of crack bridging forces." Int. J. Fracture., 49(3), 159-176.

Fett, T., Munz, D., Seidel, J., Stech, S., and Rodel, J. (1996). “Correlation between long and short crack R-curves in Alumina using crack opening displacement and fracture mechanical weight function method." J. Am. Ceram. Soc., 79(5), 1189-1196.

Goto, Y. (1971). "Cracks formed in concrete around deformed tension bars." ACI Journal Proceedings, 68(4), 244-251.

Higgins, D. D., and Bailey, J. E. (1976). "Fracture measurements on cement paste.” J. Mater. Sci., 11, 1995-2003.

Kirsch, A. (1996). An introduction to the mathematical theory of inverse problems. Springer-Verlag New York, Inc.

Kitsutaka, Y. (1997). "Fracture parameters by polylinear tension-softening analysis." $J$. Eng. Mech.- ASCE, 123(5), 444-450. 
Lou, J., Bhalerao, K., Soboyejo, A.B.O., and Soboyejo, W.O. (2003). "An investigation of fracture initiation and resistance-curve behavior in concrete." Cement Concrete Comp., 25(6):607-615.

Marshall, D. B., and Cox, B. N. (1987). "Tensile fracture of brittle matrix composites." Acta Metall., 35(11), 2607-2619.

Marshall, D. B., Cox, B. N., and Evans, A. G. (1985). "The mechanics of matrix cracking in brittle-matrix composites.” Acta Metall., 33(11), 2013-2021.

Massabo, R., Mumm D.R., and Cox, B.N. (1998). “Characterizing mode II delamination cracks in stitched composites.” Int. J. Fracture., 92(1), 1 - 38.

McMeeking, R. M., and Evans, A. G. (1990). "Matrix fatigue cracking in fiber composites." Mech. Mater., 9(3), 217-227.

Michael, N. A., and Herget, C. J. (1981). Applied algebra and functional analysis. Dover publications, New York.

Reddy, J. N. (1986), Applied functional analysis and variational methods in engineering. McGraw-Hill, Singapore.

Rodel, J., Kelly, J. F., and Lawn, B. R. (1990). "In situ measurements of bridged crack interfaces in the scanning electron microscope." J. Am. Ceram. Soc., 73(11), 3313 3318.

Saouma, V. E., Ingraffea, A. R., and Catalano, D. M. (1982). "Fracture toughness of concrete: KIC revisited.” J. Eng. Mech. Div.- ASCE, 108(EM6), 1152-1167. 
Studer, M., Pietrzyk, J., Peters, K., Botsis, J., and Giaccari, P. (2002). “Studies on bridging tractions: simultaneous bridging tractions and CODs measurements." Int. J. Fracture., 114(3), 379-399.

Tada, H., Paris, P. C. and Irwin, G. R. (1985). The stress analysis of cracks handbook. Paris Productions, St. Louis, Missouri.

Tikhonov, A. N., Goncharsky, A. V., Stepanov, V. V., and Yagola, A. G. (1990), Numerical methods for the solutions of ill-posed problems. Kluwer academic publishers group, Dordrecht, The Netherlands.

\section{Appendix A: Weight Function of an SEN Specimen}

The functions to define the weight function of a single edge notched specimen in Eq. (3) are

$$
g(x / a, \xi)=g_{1}(\xi)+\frac{x}{a} g_{2}(\xi)+\frac{x^{2}}{a^{2}} g_{3}(\xi)+\frac{x^{3}}{a^{3}} g_{4}(\xi)
$$

where

$$
\begin{aligned}
g_{1}(\xi)= & 0.46+3.06 \xi+0.84(1-\xi)^{5}+0.66 \xi^{2}(1-\xi)^{2} \\
g_{2}(\xi)= & -3.52 \xi^{2} \\
g_{3}(\xi)= & 6.17-28.22 \xi+34.54 \xi^{2}-14.39 \xi^{3}-(1-\xi)^{3 / 2} \\
\quad & -5.88(1-\xi)^{5}-2.64 \xi^{2}(1-\xi)^{2} \\
g_{4}(\xi)= & -6.63+25.16 \xi-31.04 \xi^{2}+14.41 \xi^{3}+2(1-\xi)^{3 / 2} \\
& -5.04(1-\xi)^{5}+1.98 \xi^{2}(1-\xi)^{2}
\end{aligned}
$$




\section{Appendix B: Using Sobolev Space in the Domain}

If assumption of a smooth $f^{-}(x)$ on $[0, a]$ does not yield stable solutions, existence and uniqueness can be forced by enlarging or reducing the solution space, i.e. applying more restrictions on the topology of the space $D(T) \subset Z$. For example, we assume that $f^{-}(x)$ is continuous on $[0, a]$ and has almost everywhere a derivative which is square integrable on $[0, a]$. In this case, we take $D(T) \subset W_{2}{ }^{1}[0, a]$; the linear space containing the functions along with their first derivatives that are square integrable over $[0, a]$. Approximations in the transformation is computed as

$$
\left\|T-T_{h}\right\|_{W_{2}^{1} \rightarrow L_{2}} \leq h
$$

Consequently, instead of using the metric in Eq. (9), the following norm is defined for $\mathbf{f} \in W_{2}{ }^{1}[0, a]$ to substitute in Eq. (21)

$$
\|\mathbf{f}\|^{2}=\int_{0}^{a}\left[\{f(x)\}^{2}+\left\{f^{\prime}(x)\right\}^{2}\right] d x
$$

which should be numerically approximated for substitution in Eq. (23) as

$$
\|\mathbf{f}\|=\sum_{j=1}^{p} f_{j}^{2}+\sum_{j=2}^{p}\left|\frac{f_{j}-f_{j-1}}{h_{x}}\right|^{2}
$$

The elements of the matrix $\mathbf{B}$ and vector $\mathbf{v}$ will remain unchanged and the matrix $\mathbf{C}$ will be given by 


$$
\left[\begin{array}{cccccc}
1+\frac{1}{h_{x}^{2}} & -\frac{1}{h_{x}^{2}} & 0 & \ldots & 0 & 0 \\
-\frac{1}{h_{x}^{2}} & 1+\frac{1}{h_{x}^{2}} & -\frac{1}{h_{x}^{2}} & \ldots & 0 & 0 \\
\ldots & \ldots & \ldots & \ldots & \ldots & \ldots \\
0 & 0 & 0 & \ldots & -\frac{1}{h_{x}^{2}} & 1+\frac{1}{h_{x}^{2}}
\end{array}\right]
$$

\section{Appendix C: Comparison of stress intensity factors}

This section presents two sets of stress intensity factors determined by the weight function method and by the finite element method (FEM). A unit thickness RC beam with three layers of rebars is considered. The beam is under a four-point loading condition. Rebar forces at all rebar layers are computed by the transformed section analysis. External loads as well as the computed rebar forces passing through the centroids of rebar layers are shown in Fig. 9. Only one half of the beam is shown due to symmetry of the problem, which is considered for FEM analysis.

The FEM analysis was performed by ANSYS package. The crack tip region is meshed using quarter-point 8-node quadrilateral elements. Plain strain condition is assumed with linear, elastic and isotropic material model behavior. Young's modulus of concrete was $22551 \mathrm{MPa}$ (relevant to a compressive strength of $20 \mathrm{MPa}$ ) and the Poisson's ratio was 0.2. The crack tip was considered a concentration keypoint which emanates redial lines for meshing, as shown in Fig. 9. 
Results are presented in Table 1. Stress intensity factors by the weight function method are determined by Eq. (32) and (33) with the value of the weight function $G(x, a, b)$ from Appendix A. Accuracy of the weight function method for determining stress intensity factors in cases where reinforcements provide reactive forces at the crack surfaces is demonstrated by the narrow deviations as shown in Table 1.

\section{NOTATIONS}

The Following symbols are used in this paper

$\begin{array}{ll}a & =\text { Crack length } \\ b & =\text { Total depth of the beam (width of the fracture specimen) } \\ d_{b} & =\text { Diameter of rebar } \\ E_{c} & =\text { Young's modulus of concrete } \\ F_{1}, F_{2, \ldots \ldots} & =\text { The rebar force in reinforcement layer } 1,2, \ldots . . \\ F & =\text { Total tensile force in the rebars of all layers } \\ f & =\text { The rebar force simulated as force/unit length within the diameter } \\ f^{-} & =\text {Exact vector of rebar force } \\ G & =\text { Eeight function for fracture specimens } \\ g & =\text { Step interval in discretization } \\ H & \end{array}$




\begin{tabular}{|c|c|}
\hline$K_{a}$ & $=$ Stress intensity factor due to the applied load \\
\hline$K_{b}$ & $=$ Stress intensity factor due to the rebar force \\
\hline$K_{I C}$ & $=$ Fracture toughness of concrete \\
\hline$K_{\text {tip }}$ & $=$ Net stress intensity factor at the crack tip \\
\hline$L_{2}$ & $=$ Linear space of square-integrable functions \\
\hline$M$ & $=$ Applied moment on the crack plane \\
\hline$M_{n}$ & $=$ Nominal moment capacity of the beam cross-section at crack plane \\
\hline$R$ & $=$ Field of real numbers \\
\hline$T$ & $=$ The transformation between the rebar force and the CODs \\
\hline$T_{\beta}$ & $=$ The approximated transformation. \\
\hline$U$ & $=$ Linear space where the crack closure vectors belong to \\
\hline$u$ & $=$ Crack opening displacement \\
\hline$u_{a}$ & $=$ Crack opening due to the applied force \\
\hline$u_{b}$ & $=$ Crack closing due to the rebar force \\
\hline$x$ & $=$ Location along the crack $\left(x^{\prime}, a^{\prime}\right.$ are dummy variable for $\left.x\right)$ \\
\hline$Z$ & $=$ Linear space where rebar force functions belong to \\
\hline$\alpha$ & $=$ The regularization parameter \\
\hline$\delta$ & $=$ Error in data \\
\hline$v$ & $=$ Poisson's ratio \\
\hline$\rho$ & $=$ Metric (distance between vectors within a linear vector space) \\
\hline$\sigma$ & $=$ Applied stress that would exist in the crack plane in case of no crack \\
\hline
\end{tabular}


Table 1. Comparison of mode I stress intensity factors $\left(K_{I}\right)$

\begin{tabular}{|l|l|l|l|}
\hline Crack & $K_{I}$ by the & $K_{I}$ by FEM & Deviations \\
$(\mathrm{mm})$ & analysis by & $\%$ \\
& menthetion & ANSYS & \\
$\left(\mathrm{N} / \mathrm{mm}^{3 / 2}\right)$ & \\
\hline 225 & 31.12 & 34.32 & $9.3 \%$ \\
\hline 250 & 36.26 & 39.43 & $8.0 \%$ \\
\hline 275 & 39.58 & 42.2 & $6.2 \%$ \\
\hline 300 & 41.26 & 42.84 & $3.6 \%$ \\
\hline 325 & 41.15 & 41.21 & $0.1 \%$ \\
\hline 350 & 38.82 & 37.04 & $-4.8 \%$ \\
\hline
\end{tabular}




\section{List of Figures}

Figure 1. The applied bending stress and the rebar force acting on the crack plane.

Figure 2. The rebar force is simulated by the unit step functions.

Figure 3. Deviations accounted in CODs profiles due to numerical approximations.

Figure 4. Beam cross-section considered in the first set of numerical examples.

Figure 5. Net stress intensity factors $\left(K_{t i p}\right)$ at different levels of service moments for the beam in Fig. 4.

Figure 6. CODs profiles at different levels of service moments for the beam in Fig. 4.

Figure 7. The rebar force determined by inverse analysis for the beam in Fig. 4 when $M / M_{\mathrm{n}}=0.8$.

Figure 8. A specific example (a) an RC beam on a four-point loading, (b) cross-section of the beam, (c) the rebar force obtained by Eq. (31), (d) CODs profile obtained by the direct solution of Eq. (1), (e) retrieved rebar force from CODs data with $1 \%$ error, (f) retrieved rebar force from CODs data with 5\% error.

Figure 9. Two dimensional half-span beam for FEM analysis by ANSYS package with the adopted mesh. 


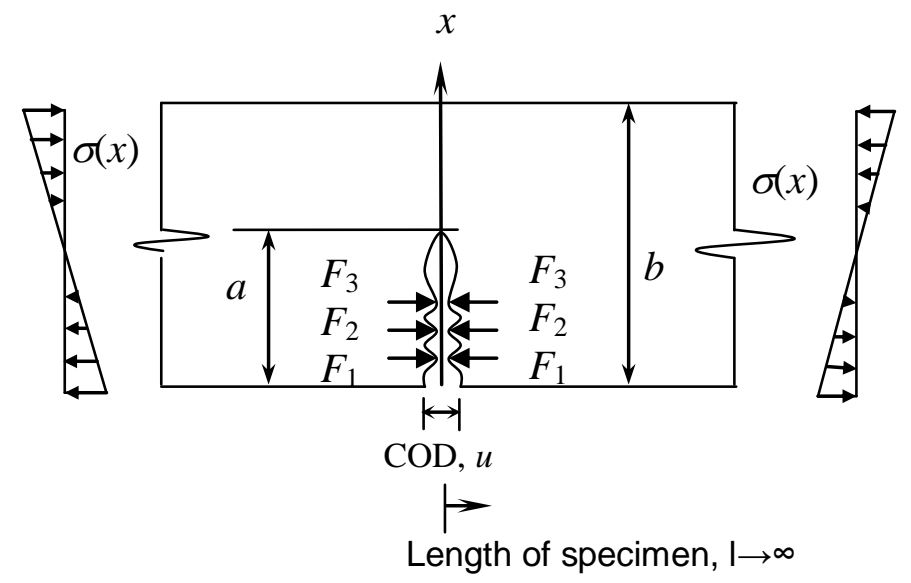

Fig. 1 The applied bending stress and the rebar force acting on the crack plane.

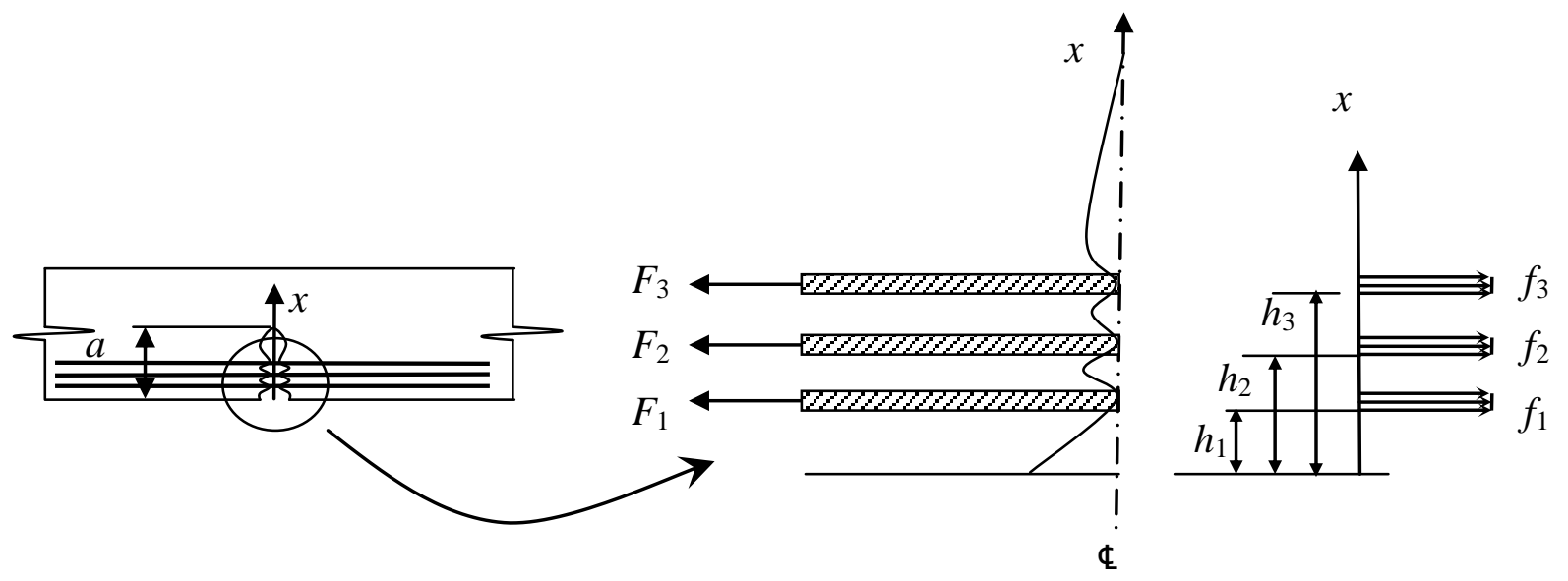

Fig. 2 The rebar force is simulated by the unit step functions. 


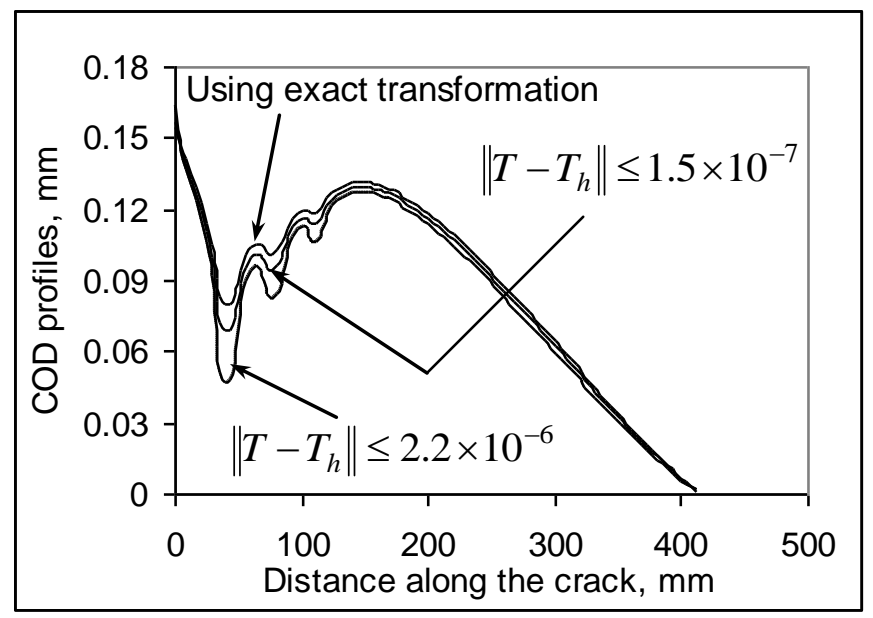

Fig. 3 Deviations accounted in CODs profiles due to numerical approximations.

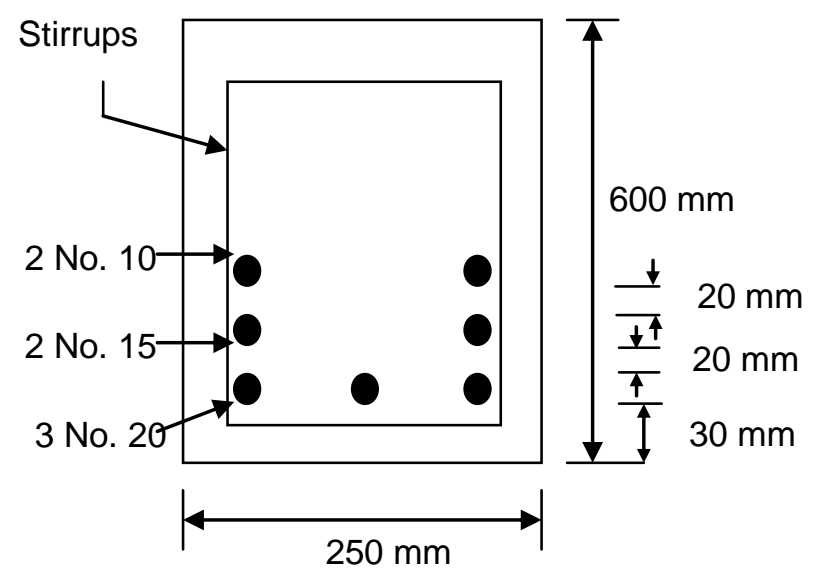

Fig. 4 Beam cross-section considered in the first set of numerical examples. 


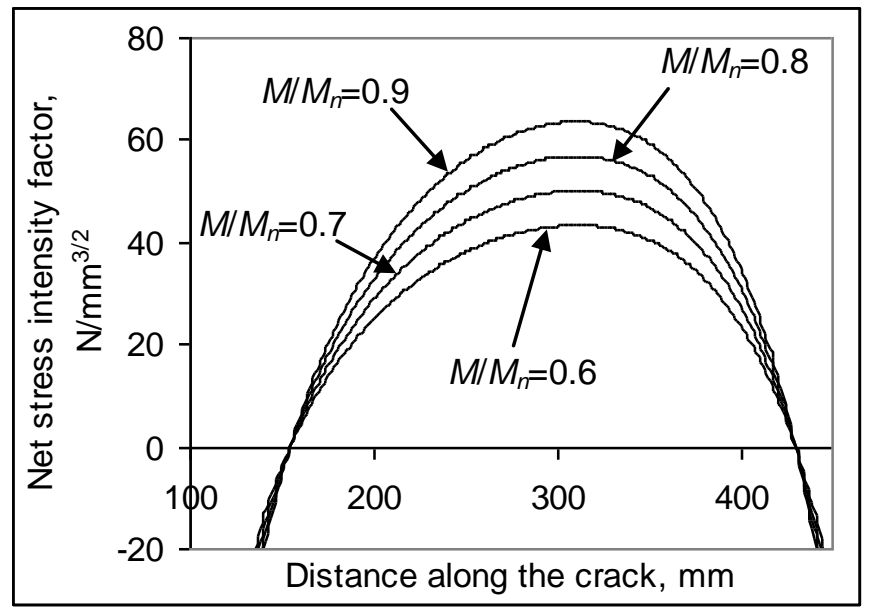

Fig. 5 Net stress intensity factors $\left(K_{t i p}\right)$ at different levels of service moments for the beam in Fig. 4.

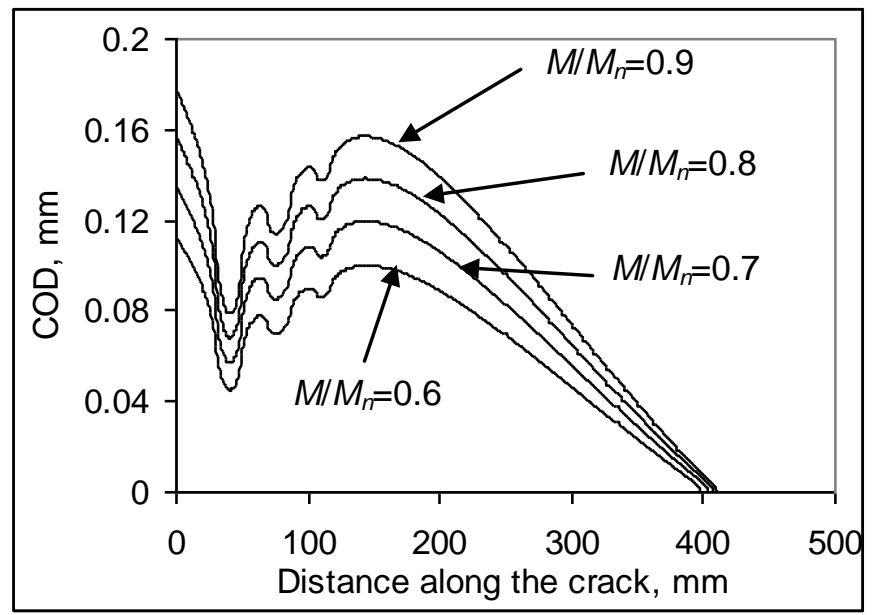

Fig. 6 CODs profiles at different levels of service moments for the beam in Fig. 4. 

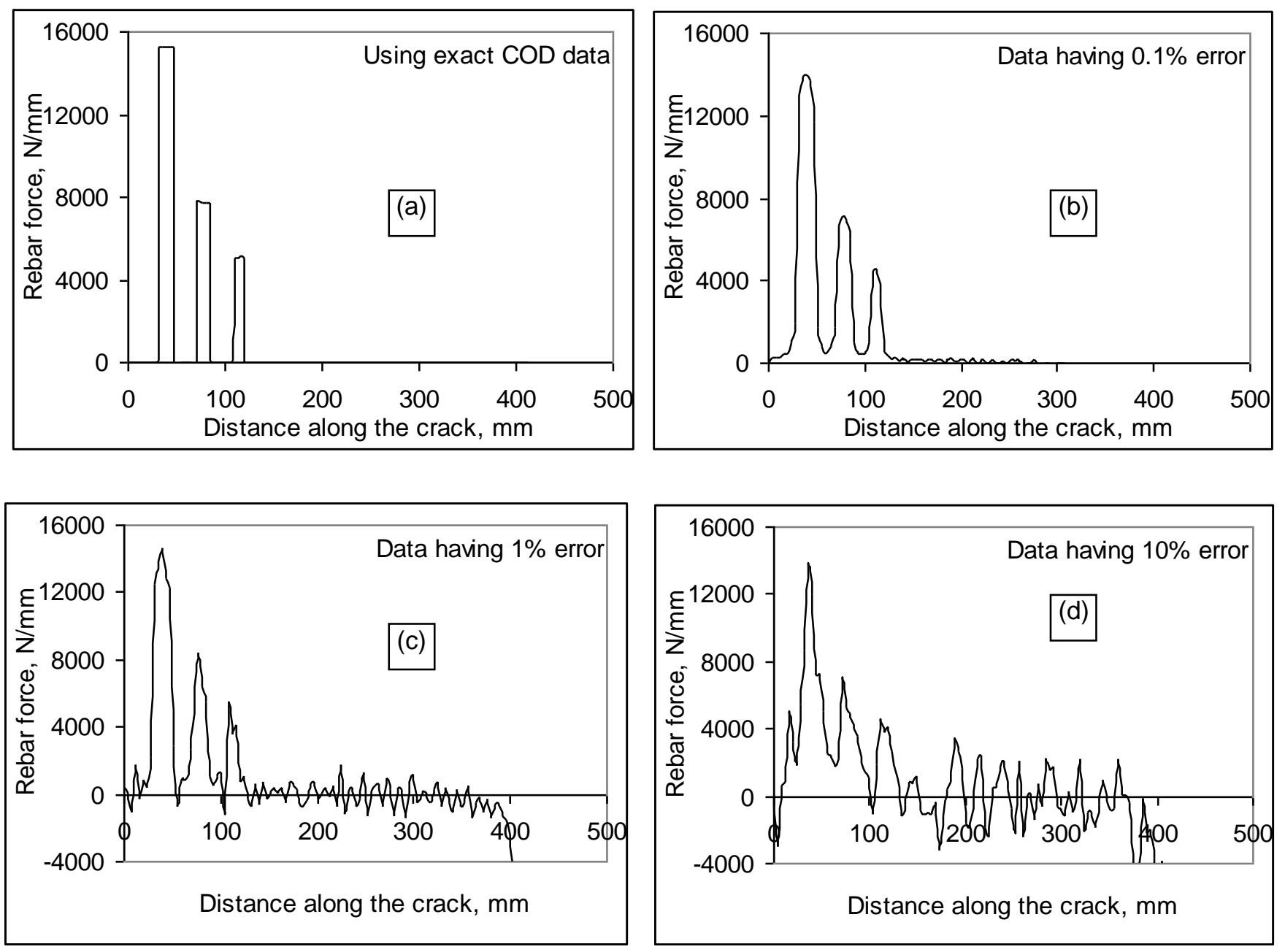

Fig. 7 The rebar force determined by inverse analysis for the beam in Fig. 4 when $M / M_{\mathrm{n}}=0.8$. 


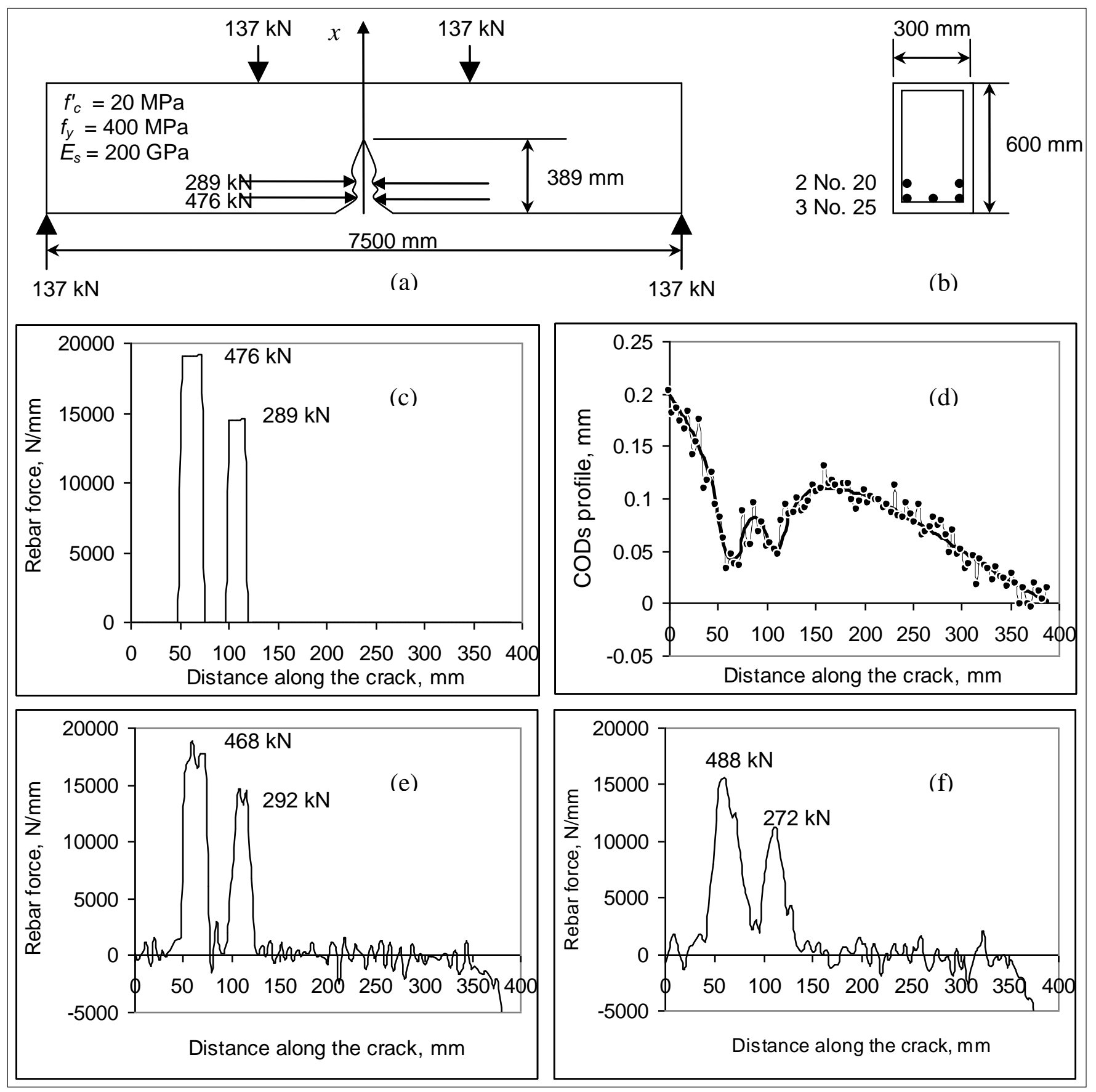

Fig. 8 A specific example (a) an RC beam on a four-point loading, (b) cross-section of the beam, (c) the rebar force obtained by Eq. (31), (d) CODs profile obtained by the direct solution of Eq. (1), (e) retrieved rebar force from CODs data with $1 \%$ error, (f) retrieved rebar force from CODs data with $5 \%$ error. 


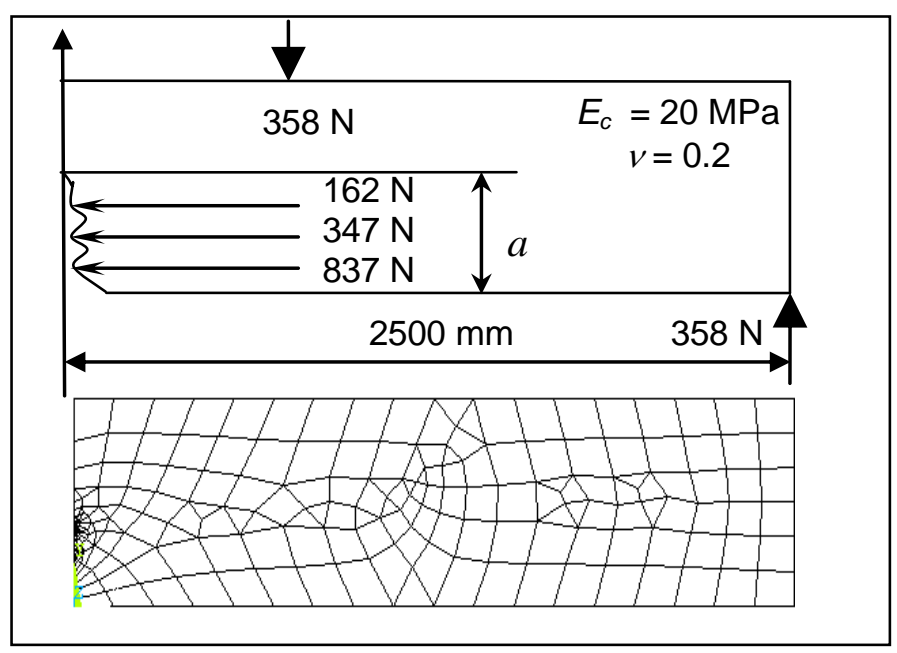

Fig. 9 Two dimensional half-span beam for FEM analysis by ANSYS package with the adopted mesh. 